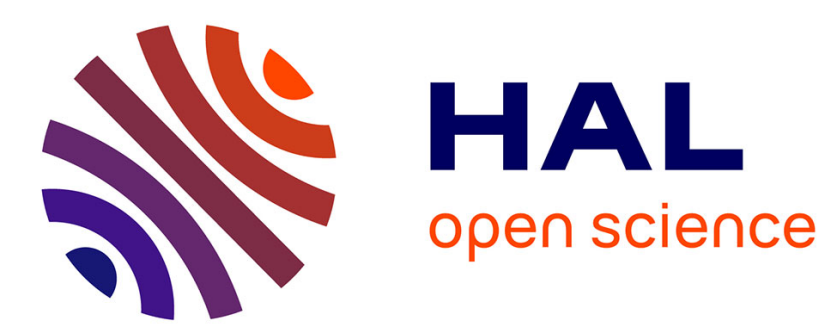

\title{
Enhancement and anticipation of the Ioffe-Regel crossover in amorphous/nanocrystalline composites
}

Ameni Tlili, Valentina M. Giordano, Yaroslav Beltukov, Paul Desmarchelier, Samy Merabia, Anne Tanguy

\section{- To cite this version:}

Ameni Tlili, Valentina M. Giordano, Yaroslav Beltukov, Paul Desmarchelier, Samy Merabia, et al.. Enhancement and anticipation of the Ioffe-Regel crossover in amorphous/nanocrystalline composites. Nanoscale, 2019, 11 (44), pp.21502-21512. 10.1039/C9NR03952J . hal-02322433

\section{HAL Id: hal-02322433 \\ https://hal.science/hal-02322433}

Submitted on 24 Nov 2020

HAL is a multi-disciplinary open access archive for the deposit and dissemination of scientific research documents, whether they are published or not. The documents may come from teaching and research institutions in France or abroad, or from public or private research centers.
L'archive ouverte pluridisciplinaire HAL, est destinée au dépôt et à la diffusion de documents scientifiques de niveau recherche, publiés ou non, émanant des établissements d'enseignement et de recherche français ou étrangers, des laboratoires publics ou privés. 


\section{Journal Name}

\section{ARTICLE TYPE}

Cite this: DOI: 10.1039/xxxxxxxxxx

Received Date

Accepted Date

DOI: $10.1039 / x x x x x x x x x x$

www.rsc.org/journalname

\section{Enhancement and anticipation of the loffe-Regel crossover in amorphous/nanocrystalline composites ${ }^{\dagger}$}

\author{
A. Tlili, ${ }^{a}$ V. M. Giordano, ${ }^{b{ }^{\ddagger}}$ Y. M. Beltukov, ${ }^{c}$ P. Desmarchelier, ${ }^{d}$ S. Merabia,${ }^{b}$ and A. \\ Tanguy $e^{e+\frac{1}{*}}$
}

\begin{abstract}
Nanocomposites made of crystalline nanoinclusions embedded in an amorphous matrix are at the forefront of current research for energy harvesting applications. However, the microscopic mechanisms leading alternatively to an effectively reduced or enhanced thermal transport still escape understanding. In this work, we present a molecular dynamics simulation study of model systems, where for the first time we combine a microscopic investigation of phonon dynamics with the macroscopic thermal conductivity calculation, for shedding light on thermal transport in these materials. We clearly show that crystalline nanoinclusions represent a novel scattering source for vibrational waves, modifying the nature of low energy vibrations and significantly anticipating the propagative to diffusive crossover (loffe-Regel), usually located at energies of few $\mathrm{THz}$ in amorphous materials. Moreover, this crossover position can be tuned by changing the elastic contrast between nanoinclusions and matrix, and anticipated by a factor as large as 10 for a harder inclusion. While the propagative contribution to thermal transport is drastically reduced, the calculated thermal conductivity is not significantly affected in the chosen system, as the diffusive contribution dominates heat transport when all phonons are thermally populated. These findings allow finally to understand the panoply of contradictory results reported on thermal transport in nanocomposites and give clear indications on the characteristics that the parent phases should have for efficiently reducing heat transport in a nanocomposite.
\end{abstract}

\section{Introduction}

Thermal management is one of the most urgent challenges arising in our modern technological society. Indeed, heat represents almost $66 \%$ of the energy waste in current processing and technologies, thus asking for concrete improvements aimed at reducing heat dissipation and waste, or at converting such heat in another form of energy 1 . In the late years, with the aim of positively answering to this challenge, scientists have focused in understanding heat transport in different kinds of materials, for ultimately controlling it and engineering novel materials able to limit heat spread and dissipation while keeping other functional properties,

\footnotetext{
${ }^{a}$ Université de Lyon, LaMCoS, INSA-Lyon, CNRS UMR5259, F-69621, France

${ }^{b}$ Institut Lumière Matière, UMR 5306 Université Lyon 1-CNRS, F-69622 Villeurbanne Cedex, France.

* Corresponding Author.valentina.giordano@univ-lyon1.fr

${ }^{c}$ Ioffe Institute, 194021 St. Petersburg, Russian Federation

d LaMCos, INSA-Lyon, CNRS UMR5259, Université de Lyon, F-69621 Villeurbanne Cedex, France

* Corresponding Author. anne.tanguy@insa-lyon.fr

$\dagger$ Electronic Supplementary Information (ESI) available: [details of any supplementary information available should be included here]. See DOI: 00.0000/00000000.
}

such as optical or electrical.

Recently, the use of composites made of guest nanoparticles embedded in a uniform host matrix has arisen as a very promising approach $^{2}$. Indeed, many theoretical and experimental studies have evidenced a strong reduction of the vibrational contribution to thermal conductivity, which has been understood in terms of an enhanced phonon scattering due to inclusions, and cannot be accounted for by standard effective medium theories, although successful in describing macroscopic composites 3 . Modified effective medium theories have been recently proposed, able to account for phonon scattering processes arising on the nanometric scale both in the matrix and within the nanoinclusions, and have succeeded in reproducing the results of simulations on the thermal conductivity of nanocomposites and its dependence on interface density 10 11. Still, even these models fail in reproducing exotic phenomena reported by numerical studies, such as the high energy phonon localization in the intergrain regions ${ }^{12}$ or their filtering through nanometric inclusions in an amorphous matrix 13 , indicating thus that the microscopic mechanisms at play still escape understanding.

Intuitively, one of the key parameters ruling the vibrational en- 
ergy transport in such materials is the contrast of elastic properties between inclusion and matrix, similar to an impedance mismatch. The higher the contrast, the stronger the interface scattering on phonons traveling in the matrix. Indeed, it has been recently reported that no remarkable effect could be observed in thermal and electric transport properties in a nanocomposite when the elastic and electric properties of the crystalline inclusions are too similar to the ones of the matrix ${ }^{14}$. When the elastic contrast is significant, instead, contradictory results have been reported, such as a strong thermal conductivity reduction, as measured in composites made of Si nanocrystals embedded in a polystyrene matrix ${ }^{9}$ or its enhancement beyond normal percolation as simulated in glassy silica with embedded nanocrystalline inclusions of GaN, both results being at odds with all effective medium predictions 15 . It is thus still not understood how the elastic contrast acts on thermal transport in such systems. To shed light on this question, it is essential to look directly at the effect of interfaces and elastic contrast onto the heat carriers, the phonons. However, until now only very few works have adopted a microscopic approach for understanding the mechanisms at play, the majority of the literature on the subject being focused on the simulation of the thermal conductivity or the interpretation of experimental data by means of effective medium models.

Here we report a molecular dynamics investigation aimed at unveiling the role of elastic contrast in amorphous/crystalline nanocomposites and getting a microscopic understanding of the effect of nanometric elastic heterogeneities and interfaces on thermal transport, through the study of phonon dynamics. Such composites, made of nanocrystalline particles embedded in an amorphous matrix, are here chosen for their high technological potential. They offer indeed the opportunity of realizing a material with a low glass-like, matrix-dominated, thermal conductivity, together with good electrical properties, as determined by the crystalline guest phase through a percolation phenomenon 2 . To this purpose, we have investigated a model 3D silicon amorphous system, with nanometric Si crystalline inclusions. The elastic contrast has been tuned by modifying the inter-atomic potential within the inclusion, in such a way that the Young modulus is the same as in the amorphous matrix in one case, and about five times larger in a second case.

It is worth reminding here that in amorphous materials, three dynamical regimes can be identified 16]17: i) a low-energy planewave dominated regime, where phonons undergo weak scattering, insuring a propagative thermal transport ii) a strong scattering regime, above the Ioffe-Regel limit, which identifies the energy at which wave-packets lifetime has been so reduced that they cannot be considered propagative plane-waves anymore and the corresponding phonons are called "diffusons"[18. This regime corresponds to a diffusive transport of vibrational energy. This definition is the one that we will use in the following when talking of "diffusive regime", "diffusive transport". The Ioffe-Regel crossover marks then the end of the propagative in favour of a diffusive thermal transport. Finally, iii) the Anderson localization regime near the mobility edge $16 / 1920$. The question arises how the presence of interfaces and elastic heterogeneties at the nanoscale affects these dynamical regimes.
We show here that phonon dynamics is strongly modified in nanocomposites with and without elastic contrast, leading to a change in the nature of low energy phonons, significantly anticipating the propagative to diffusive crossover and the Ioffe-Regel limit with respect to the parent amorphous material. Surprisingly, the calculated thermal conductivity is apparently insensitive to the elastic contrast, which can be understood in terms of a dominant diffusive thermal transport already in the amorphous matrix.

\section{Methods}

The system is made of an amorphous silicon matrix where spherical nanoholes are scooped out and filled with fcc crystalline silicon of the same density. The amorphous matrix is obtained with molecular dynamics simulations by quenching very quickly a liquid sample of silicon atoms interacting with the Tersoff potential 21 and initially equilibrated at $3500 \mathrm{~K}$. Then a quench is performed with a quenching rate of $10^{14} \mathrm{~K} / \mathrm{s}$ in the NVT ensemble at a fixed density $\rho=2.303 \mathrm{~g} / \mathrm{cm}^{3}$ with a box size of $L_{b}=60 \AA \quad(10$ 740 atoms), and periodic boundary conditions. An energy minimization is done using overdamped dynamics as soon as the tem-

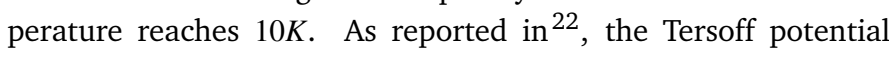
allows to get a realistic structure, whose pair correlation function and static structure factor are in agreement with experimental data, which is not the case for the Stillinger-Weber potential 23. However, this latter is better suited for the analysis of the mechanical properties 24 . For this reason, once the system generated, we replace the Tersoff potential by the StillingerWeber for our study. The system is first annealed at $100 \mathrm{~K}$ for $10 \mathrm{ps}$, and then the total energy is minimized again to get an initial amorphous matrix at mechanical equilibrium. Nanoholes with spherical shapes are then cut in the matrix and filled with nanospheres cut in a fcc silicon configuration with the same density $\rho=2.303 \mathrm{~g} / \mathrm{cm}^{3}$. In order to prevent the large stresses at the boundaries of the inclusions, atoms with neighbors at distances below 1 Åare removed, and the nano-composite is again annealed at $100 \mathrm{~K}$ for $10 \mathrm{ps}$ and quenched.This procedure allows atoms to readjust enough for avoiding instabilities initiated at the interface and getting a deeper mechanical equilibrium. Different inclusions sizes have been compared 25 . In this work, we focus on the case of an inclusion radius $R=25$ Athat was found to give rise to the largest effect on wave packets attenuation 25 . The corresponding crystalline volume fraction is thus $\phi=30 \%$ and the total number of atoms is close to $N=10740$ in the basic cell. In order to tune the elastic contrast between inclusions and matrix, we have artificially increased the rigidity of the nanoinclusions by choosing a larger value for the prefactor $\Lambda$ of the three-body term 23 in the Stillinger-Weber interaction, which is proportional to the shear modulus $\frac{16}{16}$, affecting the transverse sound velocity as $\sqrt{\Lambda}$. For silicon it is $\Lambda=21$. We have then kept this value in the matrix, and prepared an inclusion with the same value and another one with $\Lambda=100$, doubling the transverse velocity with respect to the matrix: $c_{T}(100) / c_{T}(21)=2.20$, while increasing the longitudinal only by $40 \%\left(c_{L}(100) / c_{L}(21)=1.38^{16 / 22}\right)$. Since only the impedance ratio matters for sound propagation, we introduce the rigidity ratio $\chi=E(100) / E(21)=4.6$ to designate the nanocomposite with the more rigid inclusions and $\chi=1$ to designate 


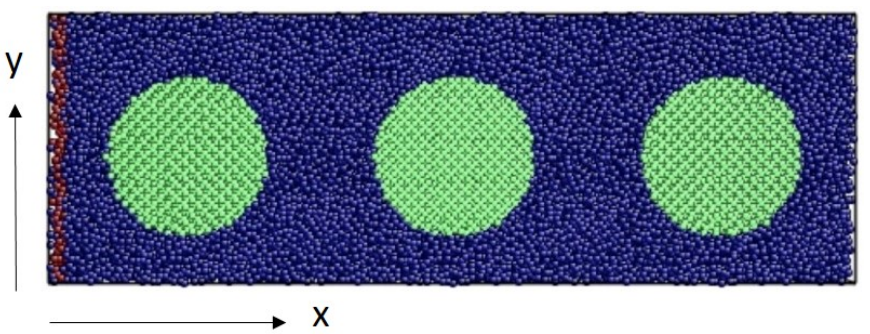

Fig. 1 Atomistic model of a nanocomposite made of an amorphous matrix (blue) and crystalline inclusions (green) with radius $R=25 \AA$ and distance between inclusions $L_{b}=60 \AA\left(L=2 L_{b}=120 \AA\right)$. The excitation of the wave packet is imposed along the red layer.

the sample with $\Lambda=21$ in the matrix and in the inclusions.

In our work, we analyze small vibrations around the equilibrium state. The properties of the near-equilibrium vibrations are determined by the properties of the dynamical matrix in the harmonic approximation

$$
M_{i \alpha, j \beta}=\frac{1}{\sqrt{m_{i} m_{j}}} \frac{\partial^{2} U}{\partial r_{i \alpha} \partial r_{j \beta}} .
$$

The numerical calculation of all second derivatives in Eq. (1) for a large atomic system is a time-consuming procedure. However, all interatomic potentials have a known analytical form, and the second derivatives can be calculated analytically. Due to the complicated structure of these latter, we use computer algebra system SymPy to find their analytical form. To generate the high-efficient numerical code from the analytical expression we use the Lambdify function. The dynamical matrix $M$ was calculated for the equilibrium state obtained by molecular dynamics. In order to calculate the DOS for the given dynamical matrix, we use the Kernel Polynomial Method (KPM) 1626 .

The dynamical structure factor $S(q, \omega)$ is obtained from the spatial and temporal Fourier Transform of the atomic displacements during a Molecular Dynamics run at constant total energy with random initial velocities on each atom. The method used to compute $S(q, \omega)$ is the same as in $\frac{13}{13}$. It allows discriminating the transverse and the longitudinal components of the dynamical structure factor. To perform the Spatial Fourier Transform, the minimum wavevector is $q_{\min }=\pi / L$ and the maximum wavevector is $q_{\max }=2 \pi /(1 \AA)$. The minimum frequency with a physical meaning is imposed by the size of the system $\omega_{\min }=c_{T} / L$. It allows defining the duration of the Molecular Dynamics run $t_{\text {run }}=2 \pi / \omega_{\min }$, while the maximum frequency is related to the inverse timestep $\Delta t=10 f s$ as $\omega_{\max }=2 \pi / \Delta t$.

In order to study the wave packets propagation, we apply a quasi-monochromatic external pulse to a thin atomic layer between inclusions. Only the harmonic response is studied here. In this case, the equation of motion can be written using the dynamical matrix $M$

$$
\ddot{u}_{i \alpha}(\omega, t)+\sum_{j \beta} M_{i \alpha, j \beta} u_{j \beta}(\omega, t)=f_{i \alpha}^{\mathrm{exc}}(\omega, t),
$$

where $\mathbf{u}_{i}=\sqrt{m}\left(\mathbf{r}_{i}-\mathbf{R}_{i}\right)$ is a scaled displacement of the $i$-th atom from the equilibrium position $\mathbf{R}_{i}$ and $M_{i \alpha, j \beta}$ is an element of the dynamical matrix $M$. The excitation force $f_{i \alpha}^{\text {exc }}(\omega, t)$ is the $\alpha$ component of a complex excitation force

$$
\mathbf{f}_{i}^{\operatorname{exc}}(\omega, t)=\mathbf{f}_{L / T} \exp \left(i \omega t-\frac{t^{2}}{2 \tau_{\mathrm{exc}}^{2}}-\frac{x_{i}^{2}}{2 w^{2}}\right) .
$$

The width of the excited layer is $w=1 \AA$ and the duration of the excitation is $\tau_{\mathrm{exc}}=0.36 \mathrm{ps}$. Such pulse duration is smaller than a typical phonon lifetime and gives a good enough frequency resolution $\delta v \sim 1 /\left(2 \pi \tau_{\mathrm{exc}}\right)=0.4 \mathrm{THz}$. The direction of the applied force is defined by $\mathbf{f}_{L / T}$, which is common for all atoms in the excited layer. The subscript $L$ (or $T$ ) indicates the wave-packet polarization in case of coherent excitation. In this case, we use $\mathbf{f}_{L}$ in $x$ direction for the longitudinal polarization and $\mathbf{f}_{T}$ in $y$ direction for the transverse one (see Fig. 1).

In order to study the wave packets propagation for different frequencies we can use the linearity of Eq. 2 and impose an initial pulse containing all the frequencies. The deconvolution of the dynamics allows then studying all frequencies using only one simulation and the Fast Fourier Transform. The details of this procedure were described in Ref. ${ }^{27}$. The diffusivity can also be computed, by measuring the time dependence of the progressive enlargement of the wave packet with initial random polarizations. The diffusivity $D(\omega)$ is then obtained as the slope in the linear time dependence of the quantity

$$
\frac{1}{2} \frac{\sum_{i}\left(E_{k}(i, t) \times x_{i}^{2}\right)}{\sum_{i} E_{k}(i, t)}-\frac{1}{2}\left(\frac{\sum_{i} x_{i} E_{k}(i, t)}{\sum_{i} E_{k}(i, t)}\right)^{2}=\frac{1}{2}<\left(x^{2}-<x>^{2}\right)>\propto D t
$$

where $E_{k}(i, t)$ is the kinetic energy averaged on the slice located at $x_{i}$ with width $\delta x=2 \AA$.

For better averaging, we use first a system with 8 inclusions in a periodic box $L \times L \times L$ ( 2 inclusions along each side of the cube), for a total number of atoms 8 times bigger than in the basic cell. There are 6 possible planes between inclusions $(x=0$, $x=L / 2=L_{b}, y=0, y=L / 2=L_{b}, z=0$, and $z=L / 2=L_{b}$ ). We sequentially use all these planes as the excitation layers and average the results over them. Using periodic boundary conditions, this system is repeated a sufficient number of times in the direction perpendicular to the excitation plane in order to prevent the reflection of waves at the boundary during the duration of the simulation.

Finally, we computed the nanocomposite thermal conductivity $k_{T}$ using equilibrium simulations $28 \mid 31$. The thermal conductivity has been calculated by the Green-Kubo formula :

$$
k_{T}=\frac{1}{3 V k_{B} T} \int_{0}^{+\infty}\left\langle J_{\alpha}(t) J_{\alpha}(0)\right\rangle d t
$$

where $V$ is the nanocomposite volume, $k_{B}$ is Boltzmann's constant, $J_{\alpha}(t)$ is the instantaneous heat flux vector ${ }^{30}$ (Einstein summation convention has been implictly assumed), and the brackets indicate an ensemble average. NVE simulations (duration 0.25 ns) have been run to sample the heat flux vector every 10 time steps (time step $=0.5 \mathrm{fs}$ ). To obtain good convergence of the integral of Eq. 5. we have averaged the results over 10 indepen- 

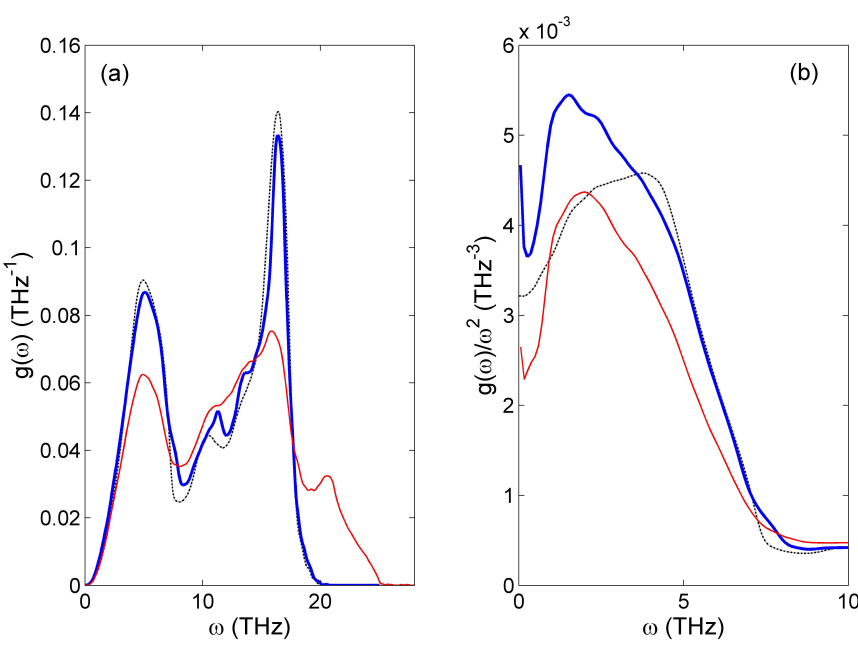

Fig. 2 Panel (a): vibrational density of states for the nanocomposite with $\chi=1$ (thick blue line), $\chi=4.6$ (thin red line) with $30 \%$ crystalline volume fraction, and for a fully amorphous sample from Ref ${ }^{16}$ (dotted black line). While in case of no contrast the DOS is only weakly affected by the presence of the inclusion, the harder inclusion introduces high energy vibrational modes. Panel (b): reduced density of states, normalized by the Debye behavior $\left(\omega^{2}\right)$ to evidence the Boson Peak at low energy. Same colors as in panel (a) for the three systems. The presence of the inclusion causes the growing up of a peak at about $1.7 \mathrm{THz}$.

dent simulations, for each studied structure. In this way, while the simulation time is large enough for the thermal conductivity calculation to converge, the error bar is reduced through the averaging over independent simulations rather than a longer simulation time, which could result in large numerical uncertainties 32 33 (see Supplementary Materials for more details). This calculation has been done using the same atomic configurations as for the microscopic dynamics simulations, on a single cell with a single inclusion and periodic conditions, with initial equilibrations at 10 $\mathrm{K}$ and also at $300 \mathrm{~K}$.

Before closing this section, it is worth commenting on the choice of considering only harmonic interactions. Indeed, anharmonicity has quite a relevant role in determining thermal transport properties at high temperature. Still, it was shown previously that it is negligible in amorphous samples at the phonon energies here concerned ${ }^{34}$, suggesting thus that the same is true in nanocomposites with a dominant amorphous component. Concerning all our results from the atomic structure equilibrated at $10 \mathrm{~K}$, we can safely rule out an anharmonic contribution in the crystalline component as well, and thus in nanocomposites whatever the crystalline content. The question arises concerning the calculation of the thermal conductivity from the configuration equilibrated at $300 \mathrm{~K}$. We will comment on this point later in the paper.

\section{3 results}

\subsection{Density of States and Dynamical Structure Factor}

We report in Fig. 2 panel (a) the total vibrational density of states (DOS) as calculated in the samples with and without elastic contrast, together with the DOS of a pure amorphous sample, from
Ref.16. The $\chi=1$ system presents some differences from the fully amorphous sample, mostly localized at about 11 and $13.3 \mathrm{THz}$, where the DOS is sharper, reminiscent of sharper features in the crystalline system. As for the $\chi=4.6$ sample, new high energy modes appear in the density of states for $\omega \geq 20 \mathrm{THz}$, characteristic of the harder inclusion. The number of modes in the $\omega \leq 20 \mathrm{THz}$ region obviously decreases because of the normalization of the DOS, still the relative weight of the modes at around $10 \mathrm{THz}$ becomes more important.

To better inspect the low energy acoustic modes, we report in panel (b) the reduced density of states, normalized to the Debye behavior, where the characteristic excess of low energy modes, the Boson Peak, clearly appears in the amorphous system at $\approx 4 \mathrm{THz}$. Interestingly, in both nanocomposites, this region is strongly affected, leading to the growing up of a low-frequency peak, at about $1.7 \mathrm{THz}$, already weakly present in the fully amorphous system. As such, the DOS of the nanocomposite reminds of the one calculated in ${ }^{16}$ for a weak three-body interaction (parameter $\lambda<21$ in the three-body term of the inter-atomic potential). This low energy peak was identified in that work as corresponding to the first strong minimum of the atomic participation ratio, i.e. the end of the plane-wave, Debye-like, regime, prior to the reaching of the Ioffe-Regel transition from propagative to fully scattered waves. In that work the low energy peak corresponds to a typical distance between scatterers of $\approx 20 \AA$, which is comparable here to the interparticle distance. Our results point thus to a larger number of modes implied by the breaking down of the Debye approximation in both nanocomposites.

To understand how the presence of the nanoinclusions modifies the individual modes, we have calculated the dynamical structure factor $S(q, \omega)$, as described in section 2 . Similarly to what was done in Damart et al. 13 , to overcome the resolution limitation of the simulation, we have convoluted the $S(q, \omega)$ with a typical energy resolution function with a linewidth of $\approx 1.4 \mathrm{meV}$. The result of such data treatment is reported in Fig. 3 for modes with an exchanged momentum smaller than $0.7 \AA^{-1}$. Like in Damart's work, we also find a nice single peak at low-momentum transfers, which evolves into an envelope of many modes at higher $q$. However, while the behavior of the sample $\chi=1$ closely resembles the one of the amorphous matrix in Damart et al., in the sample $\chi=4.6$ this remains true only for the transverse polarization, while the breaking down of the single phonon in an envelope of modes takes place much earlier for the longitudinal polarization.

In order to get a more quantitative understanding, we have fitted these data using a Lorentzian function convoluted with the instrumental resolution. Where the peak was not single anymore, a fit with multiple modes has been done. In Fig. 4 we report the longitudinal and transverse acoustic dispersion obtained in this way for the nanocomposites, compared with the ones of amorphous silicon obtained by Damart et al., using the same simulation technique as well as analysis method 13 . Where the peak becomes an envelope, only the center of mass of the envelope has been reported. Here it can be seen that the acoustic dispersion is not affected by the presence of a nanocrystalline inclusion when there is no elastic contrast, in agreement with what previ- 

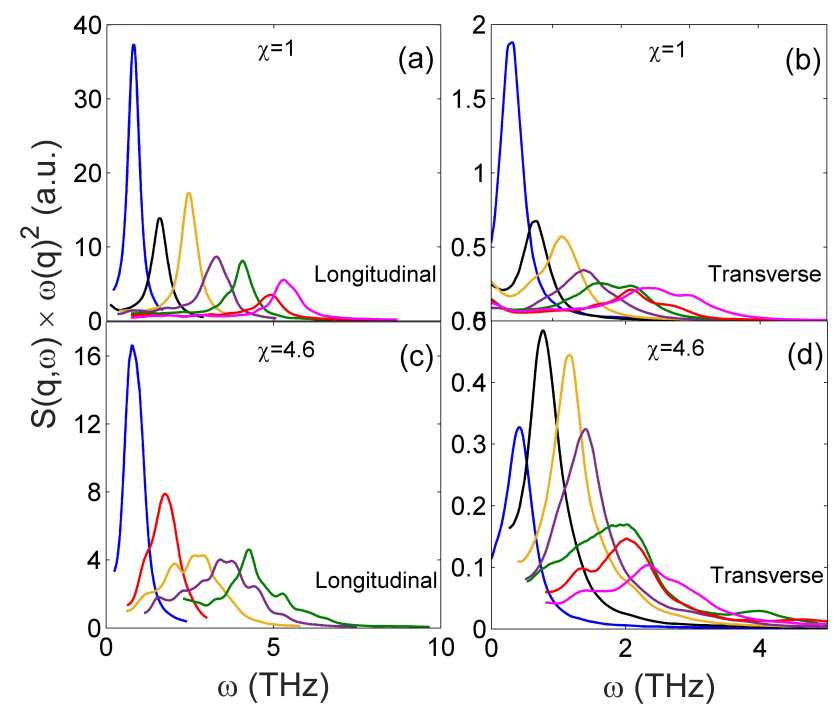

Fig. 3 Panels (a)-(b) and (c)-(d) represent the longitudinal and transverse $S(q, \omega)$ calculated in the system without elastic contrast and with elastic contrast respectively. The intensity has been multiplied by the square of the phonon energy position for enhancing the high q modes. Different colors correspond to different wave-vectors: $q=0.1$ (blue), 0.2 (black), 0.3 (orange), 0.4 (purple), 0.5 (green), 0.6 (red), 0.7 (violet) $\AA^{-1}$. The breaking down of the phonon in an envelope of modes and loss of total intensity clearly appear with the presence of an elastic contrast.
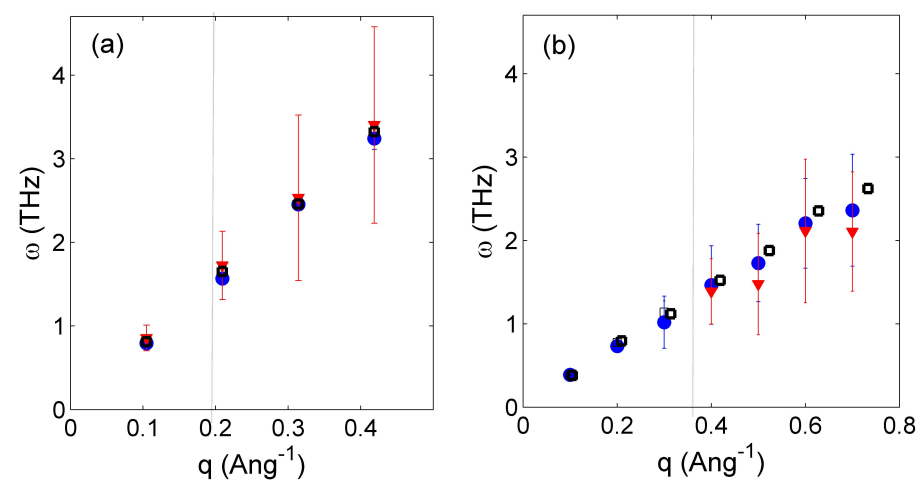

Fig. 4 (a) and (b) report the phonons acoustic dispersion respectively for the longitudinal and transverse vibrations, up to the largest $q$ at which an envelope was still visible for the systems $\chi=1$ (blue filled circles) and $\chi=4.6$ (red filled triangles). Data from Damart et al. $\frac{13}{13}$ for the fully amorphous system are also reported as black squares. The vertical dotted line separates the low q region, where a single mode exists, from the high q region, where the mode breaks in an envelope of modes. In the single mode region, the symbols correspond to the phonon energy and their error bar to its broadening, inversely proportional to its lifetime. In the envelope-region, the symbols correspond to the envelope center of mass, and the error bar to its broadening, i.e. to the width of the distribution of modes in which the phonon breaks down. ously reported and with our results on the DOS. Interestingly, in the $\chi=4.6$ sample, despite the different velocities of the modes within the envelope, the center of mass closely follows the acoustic dispersion in the pure amorphous, indicating that the dynamics is globally dominated by the matrix at these wavelengths. This result is at odds with a prediction based on the Reuss approximation for the homogenized calculation of the moduli (see Ref. 35 .36 for the definition), which works well for macroscopic composites with spherical inclusions. This approximation leads in the long wavelength limit to an effective longitudinal or shear modulus $M_{e f f}=\left(\frac{x}{M_{c}}+\frac{1-x}{M_{a}}\right)^{-1}$, with subscript $a$ or $c$ for amorphous and crystalline phase, and $x$ the crystalline fraction. Calling $v_{L / T}^{a}$ the longitudinal/transverse velocity of the amorphous matrix, for $\chi=4.6$ and $\mathrm{x}=30 \%$, we should find $v_{L}=1.11 v_{L}^{a}$ and $v_{T}=1.14 v_{T}^{a}$. Such increase is obviously not reproduced by the center of mass of the envelop, pointing thus to a failure of the effective medium approach for the description of the dynamic elastic properties in our nanocomposites at lengthscales smaller than $\approx 6 \mathrm{~nm}$.

In Fig. 4 we report as error bars the phonon broadening when a single peak exists, and the envelope full width at half maximum otherwise. From these results, we can safely conclude that the presence of a harder nanoinclusion hinders the phonon propagation, the phonon breaking down in an envelope of modes getting broader and broader as the average energy and wavevector increase.

It is worth reminding here that in pure amorphous silicon $\frac{16}{}$, the Ioffe-Regel limit is located at $4.5 \mathrm{THz}$ and $12.7 \mathrm{THz}$ for transverse and longitudinal waves respectively, for an exchanged momentum of 0.73 and $1.0 \AA^{-1}$. The vibrational modes here reported lie thus all in the propagative regime, suggesting that the presence of hard nanoinclusions induces an enhanced diffusive character of phonons, with the anticipation of the Ioffe-Regel criterion validity to lower energies. Interestingly, the breaking down of the longitudinal phonon shown in Fig. 4(a), while starting at $q \sim 0.2 \AA^{-1}$, takes clearly place at $q=0.3 \AA^{-1}$, i.e. at a wavelength of $\approx 21 \AA$, comparable with the inter-particle distance.

\subsection{Wave-packets propagation}

In order to better understand and confirm the possible change in the nature of the low energy vibrational modes induced by the presence of a harder inclusion, we have followed the propagation of longitudinal and transverse wave-packets generated in the center of the sample, as explained in section 2, Our results are directly comparable with the ones reported in 27 , for a fully amorphous silicon system.

Following Ref. 27 , we calculate the kinetic energy density as a function of space and time, averaged over an atomic plane perpendicular to the wave-packet propagation, of dimensions $L^{2}$, where $L$ is the simulation box side:

$$
E_{L / T}(\omega, x, t)=\frac{1}{2 L^{2}} \sum_{i}\left|\dot{u}_{i}^{L / T}(t)\right|^{2} \delta\left(x-x_{i}\right) ;
$$

where the sum runs over all the atoms $i$ with longitudinal or transverse velocity $\dot{u}_{i}^{L / T}$. The space and time behavior of $E_{L / T}$ for a given frequency $\omega$ allows to understand the propagative or 


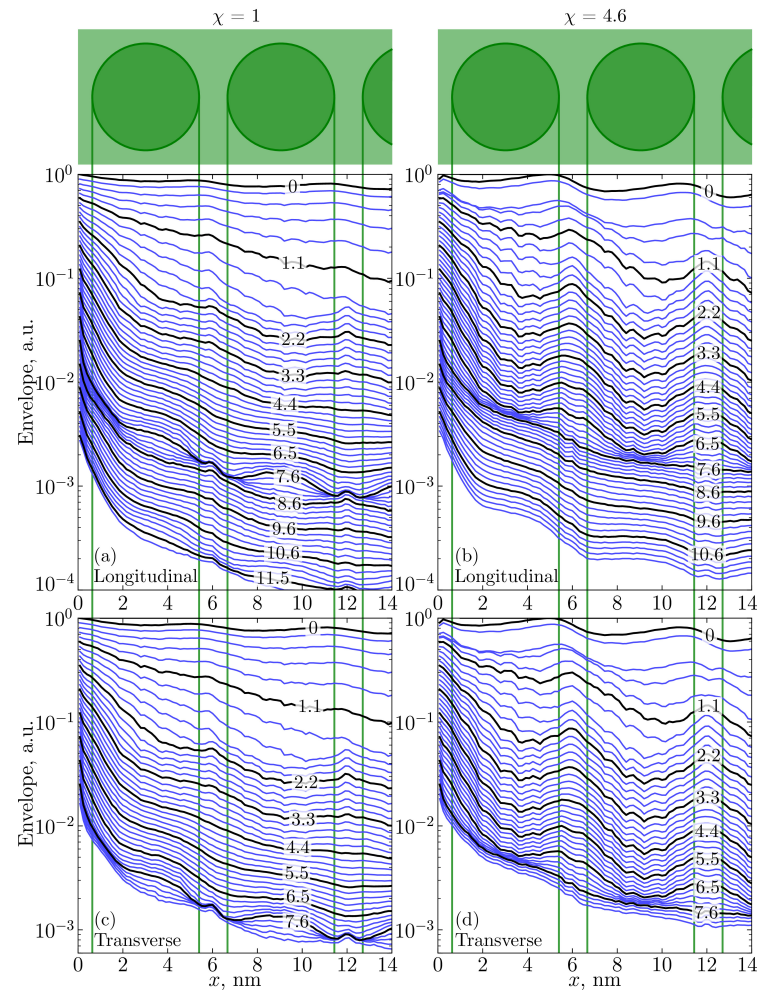

Fig. 5 The Longitudinal $(a, b)$ and Transverse (c,d) kinetic energy envelope, as calculated in $\mathrm{Eq} 7$ is reported for the system with no elastic contrast $(\chi=1)$ on the left, and with elastic contrast $(\chi=4.6)$ on the right, for different frequencies. Each fifth curve is reported as a thicker black line, and its frequency is indicated $(\mathrm{THz})$. Each $\mathrm{n}$-th curve is multiplied by $0.9^{n}$ to make an offset along the $y$-axis for a better visualisation.

diffusive nature of the wave-packet of that frequency. A very convenient way for visualizing it consists in calculating the kinetic energy envelope, by taking the maxima of the kinetic energy density at all times, thus getting rid of the time dependence:

$$
P_{L / T}(\omega, x)=\max _{t}\left[E_{L / T}(\omega, t, x)\right] ;
$$

Depending on the regime (propagative or diffusive), the envelope has different spacial dependencies, ranging from an exponential dependence similar to the Beer-Lambert law for a propagative wave-packet $\frac{37}{37}$ to an hyperbolic dependence for a diffusive wave-packet:

$$
\begin{gathered}
P_{L / T}^{p r o p}=\frac{\varepsilon_{0}}{\sqrt{\pi v^{2} \tau_{e x c}^{2}}} e^{\frac{-x}{I}} ; \\
P_{L / T}^{d i f f}=\frac{\varepsilon_{0}}{\sqrt{2 \pi e}} \frac{1}{x} ;
\end{gathered}
$$

For the derivation of these dependencies, we address the reader to Ref. 27 . Here we just say that $\varepsilon_{0}$ represents the total kinetic energy delivered to the system per unit surface from the excitation force, $\tau_{e x c}$ is the excitation duration time, and $v$ is the sound velocity with which the wave-packet moves in the propagative regime.

Figure 5 reports the envelopes for longitudinal and transverse polarization respectively, and for the two systems, for different frequencies $\omega$. It is worth underlying that the curves should be considered only for $x \geq v_{L / T} \tau_{e x c}$, due to the finite excitation time

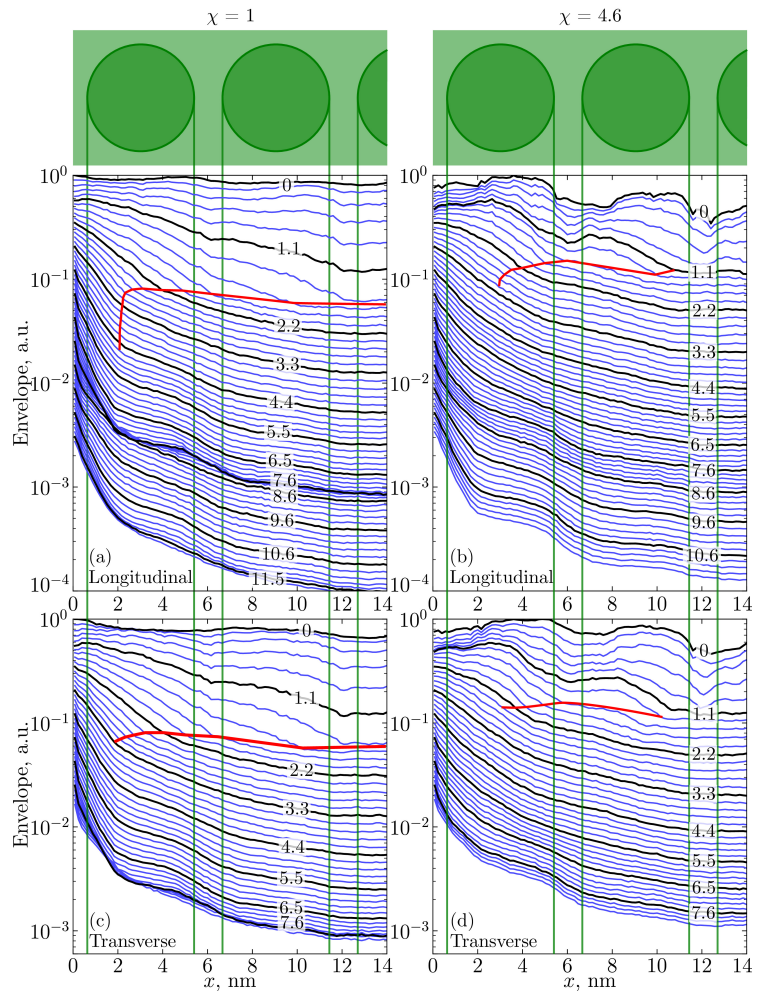

Fig. 6 Same as in Fig. 5 but after normalization by the local density of states. Thick red (grey) lines show the transition from a propagative to a diffusive regime.

$\tau_{e x c}$, i.e. $x \geq 2.8 \mathrm{~nm}$ and $x \geq 1.4 \mathrm{~nm}$ for the longitudinal and transverse polarizations respectively 13 . We have chosen here a representation in a logarithmic scale, where the Beer-Lambert law observed in the propagative regime is a straight line.

From the figure, a perfectly linear - and thus propagative - behavior is not easily found, because of the presence, in both systems, of periodic oscillations in the envelope, more evident in the case of a strong elastic contrast. These bumps present a period of $6 \mathrm{~nm}=L_{b}$ i.e. the distance between inclusions. They can be associated to the enhancement of the local density of states in correspondence to the crystalline inclusions. Indeed, the oscillations are almost fully removed if we normalize the energy envelope by the spatially resolved density of states (see Supplementary Material), as reported in Figure 6. Only at low frequency and high elastic contrast, the oscillation remains after normalization, with a maximum energy located now inside the inclusions. The propagation of energy is thus inhomogeneous at low frequency, due to the different density of states in the matrix and in the inclusions, and an additional slight pinning of the normalized vibrational energy within the inclusions.

In Fig. 6 a red thick line identifies the $\mathrm{x}$ position at which the envelope changes from an exponential to a $1 / x$ behavior, marking thus the traveled distance after which the wave-packet looses its propagative character and turns to a diffusive motion. Comparing with fully amorphous Silicon 27 , we find that already in the system with no contrast, $\chi=1$, the diffusive regime fully replaces the propagative one much earlier, pointing to a Ioffe-Regel cross-over located at $3 \mathrm{THz}$ for the transverse mode and $4.2 \mathrm{THz}$ for the 


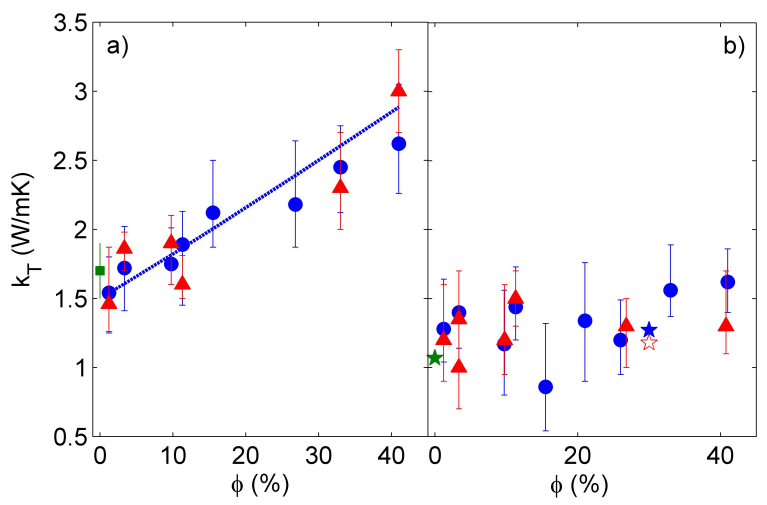

Fig. 7 Thermal conductivity calculated with the Green Kubo method in the amorphous system and the nanocomposites as a function of the crystalline volume fraction $\phi$ at $300 \mathrm{~K}(\mathrm{a})$ and $10 \mathrm{~K}(\mathrm{~b})$. Amorphous: green square, $\chi=1$ : blue circles, $\chi=4.6$ : red triangles. In (a) the EMT prediction is reported as a dashed line. In (b) we report also the thermal conductivity calculated starting from microscopic dynamics for the amorphous (green filled star) and the nanocomposites with $\phi=30 \%$ and $\chi=1$ (blue filled star) and $\chi=4.6$ (red empty star).

longitudinal one, against 4.5 and $12.7 \mathrm{THz}$ in the fully amorphous system.

Looking now to the $\chi=4.6$ system, the crossover is less distinguishable, so the transition line is much shorter. At low frequency the energy oscillations dominate the behavior: here the wave-packet is strongly scattered by the inclusions, which gives rise to an energy localization between them. It is important to specify that this is not a localization in the sense of the Anderson definition: by localization here we merely mean that there is a majority of kinetic energy between the inclusions and a minority within them, due to the lower density of vibrational states inside the hard inclusions at low frequencies. The propagative to diffusive regime crossover is now definitely anticipated, the diffusive regime taking it over at 2.2 and $3 \mathrm{THz}$ for transverse and longitudinal wave-packets respectively. The phase space for propagative modes is thus reduced by a factor of 2 for transverse vibrations and 4 for the longitudinal ones, suggesting a strong thermal conductivity reduction in this system. This would indeed be the case if most of the heat were carried by the propagative acoustic modes, as in crystalline materials.

\section{Thermal conductivity}

In order to understand the effects of an anticipated Ioffe-Regel crossover on thermal transport, we have calculated the thermal conductivity at room temperature for both our nanocomposites as a function of the inclusion volume fraction, $\phi=\frac{V_{i}}{L^{3}}$, with $V_{i}$ the total volume filled by the inclusions and $L^{3}$ the simulation box size.

Results are reported in Fig. 7 First of all, we notice that the value obtained for the fully amorphous sample at $300 \mathrm{~K}$, $k_{T}^{a m}=1.7 \pm 0.2 \mathrm{~W} / \mathrm{mK}$, is in good agreement with literature 38139 for such system sizes 40 . As for the composites, two points are here to be commented: the volume fraction and the elastic contrast dependence. At $300 \mathrm{~K}$, the thermal conductivity in the nanocomposites increases with the crystalline volume fraction, which can be expected due to the introduction of larger and larger well-conducting crystalline regions when the interface thermal resistance (Kapitza resitance) is negligible. The behaviour can be perfectly reproduced with an effective medium model, assuming for the fully nano-crystalline sample a thermal conductivity of $4 \mathrm{~W} / \mathrm{mK}$, as found in Ref ${ }^{41}$ for a grain size of $5 \mathrm{~nm}$, and for the amorphous phase $k_{T}=1.5 \mathrm{~W} / \mathrm{mK}$. Our result is thus quite different from the case of nanocrystalline inclusions of $\mathrm{GaN}$ embedded in amorphous $\mathrm{SiO}_{2}$, where an enhanced percolation was found 15 . More intriguing is the elastic contrast dependence: surprisingly, we don't see any effect of the elastic contrast within our error bars, although we have shown that the vibrational dynamics much depends on it.

A possible reason for this discrepancy is that our findings on the microscopic dynamics have been obtained at very low temperature. It is thus possible that the situation is different at high temperature. In order to understand thermal transport in such composites, we have thus calculated the thermal conductivity at $10 \mathrm{~K}$, so that it is directly related to the observed microscopic dynamics. Results are also reported in Fig.7 7 Here we see that now the thermal conductivity does not depend anymore on the crystalline volume fraction. Such a different behavior with respect to $300 \mathrm{~K}$ can be understood in terms of a temperature dependent equilibrium between the interface scattering process, which decreases the total thermal conductivity, and increases with the interface area, the addition of more conductive regions, which increases with the crystalline volume, and the anharmonic processes, which, if negligible at $10 \mathrm{~K}$, are clearly important at $300 \mathrm{~K}$. Indeed, the interface Kapitza resistance is a decreasing function of temperature ${ }^{31}$. Its larger importance at low temperature can be identified as the reason of the lack of a crystalline volume dependence of the thermal conductivity, due to a lucky compensation between interface scattering and the growing crystalline contribution. At high temperature, the latter seems to dominate on the former. Concerning the anharmonic contribution, one quick comparison of the thermal conductivity values at 10 and $300 \mathrm{~K}$ for a given crystalline volume fraction indicates that the thermal conductivity increases with temperature, which is not expected when anharmonic scattering dominates phonon lifetime. To clarify the role of anharmonicity, a thorough investigation of the thermal conductivity as a function of both temperature and elastic contrast in both amorphous and crystalline components of the nanocomposite is needed. This is however beyond the scope of this work, and makes the object of an on-going investigation. More intriguing is the fact that, at low temperature as well, the elastic contrast does not play any role, meaning that the strong reduction of the propagative regime with the elastic contrast does not reflect into a lower thermal conductivity. This could be understood in terms of a dominant role of diffusons in thermal transport already in the fully amorphous sample $\mathrm{e}^{39}$, so that the reduction of the propagative regime would be negligible.

In presence of a diffusive transport mechanism, i.e. a transport assured by largely scattered vibrational modes (namely diffusons), the thermal conductivity can be calculated knowing the phonon diffusivity, which we report in Fig. 8 for the two systems with and without elastic contrast. First, we can see here that the 


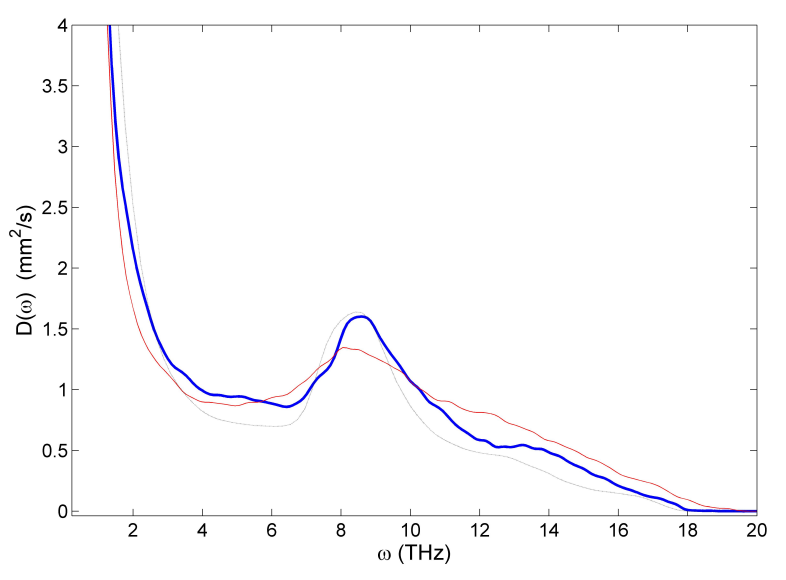

Fig. 8 The phonon diffusivity is reported between 0 and $20 \mathrm{THz}$ for the $\chi=1$ system (thick blue line) and the $\chi=4.6$ system (thin red line), compared with a pure amorphous sample (black dotted line). The high frequency modes in the $\chi=4.6$ composite, with $\omega \geq 20 \mathrm{THz}$ have negligible diffusivity.

high energy modes that appear for $\omega \geq 20 \mathrm{THz}$ have a negligible diffusivity, due to their localized nature $18 \mid 2027$, thus do not contribute to thermal transport. Second, for the other modes, the elastic contrast introduces only tiny differences, mostly reducing the diffusivity of modes around $10 \mathrm{THz}$, which, in the amorphous, comes mainly from longitudinal modes 27 . Such a weak effect may thus explain why our simulated thermal conductivity is quite insensitive to the elastic contrast.

The situation would be different if diffusons were not the dominant heat carriers. To check this point, we have calculated the thermal conductivity of a crystal/crystal nanocomposite, where the crystalline inclusions, of same radius and volume content, are now embedded in a crystalline matrix, using the same potential and rigidity contrast as before. The nanoinclusions have been tilted by $45^{\circ}$ with respect to the crystalline matrix, for maximizing the interface effect. Indeed, the resulting thermal conductivity will depend on the transmission probability of phonons from the matrix to the inclusion and viceversa, which depends on the superposition of the DOS projected along the crystallographic directions of the contact surfaces 42 43. In such systems we find $k_{T}=3.55 \pm 0.15 \mathrm{~W} / \mathrm{mK}$ and $k_{T}=2.2 \pm 0.4 \mathrm{~W} / \mathrm{mK}$ at $300 \mathrm{~K}$, for $\chi=1$ and $\chi=4.6$ respectively. We thus see a significant dependence on the elastic contrast, with a decrease by $40 \%$ of the thermal conductivity at room temperature in presence of a harder inclusion, compatible with a perturbation of the propagative acoustic modes.

To go further, and better link macroscopic and microscopic information, we have used our phonon dispersions, mean free path and diffusivity results for directly calculating the thermal conductivity. Two different regimes are considered: the propagative regime only below the Ioffe-Regel energy, and the diffusive regime, corresponding to a multiple scattering of plane waves, which remains the only one above the Ioffe-Regel energy. In the propagative regime, the thermal conductivity can be calculated as

$$
k_{T}^{\text {prop }}=\frac{1}{3} \int_{0}^{\omega_{I R}} C(T, \omega) v(\omega) l(\omega) g(\omega) d \omega
$$

Here $v(\omega)$ is the group velocity calculated from the simulated phonon dispersions fitted to a sinusoidal q dependence, in the energy region where an individual phonon mode is well defined, $C(T, \omega)$ is the quantum heat capacity, calculated from phonon dispersions and density of states, $l(\omega)$ is the mean-free path obtained by the Beer-Lambert fit of the kinetic energy envelope in the wave-packet propagation study (see Eqq.7and 8), reported in the Supplementary Material, and $g(\omega)$ the simulated density of vibrational states. In this calculation, we have taken a lower energy limit for the integral of $0.5 \mathrm{THz}$, for convergence issues, which implies that the propagative contribution is slightly underestimated. Such approximation remains reasonable as the neglected modes, despite a very long mean free path, have low heat capacity and density of states. In the diffusive regime, the thermal conductivity expression becomes:

$$
k_{T}^{\text {diff }}=\int_{\omega_{I R}}^{\omega_{\max }} C(T, \omega) D(\omega) g(\omega) d \omega
$$

with $D(\omega)$ the diffusivity of vibrational modes with energy $\omega$. Eq 10 has been calculated as the sum of the longitudinal and transverse contributions, each having a different Ioffe-Regel limit and different mean free path. To this aim, we have calculated the projected longitudinal and transverse density of states using Voronoi cells (see Supplementary Material). The total thermal conductivity is then $k_{T}=k_{T}^{\text {prop }}+k_{T}^{\text {diff }}$. In this calculation, assuming that the $S(q, \omega)$ does not change with temperature, the temperature dependence of the calculated $k_{T}$ only results from the one of the Bose factor, i.e. from the increased phonon population. As such, the thermal conductivity calculated this way at high temperature, when all phonons are thermally populated, corresponds to the thermal conductivity as calculated with the Green-Kubo method, where all phonons are always populated. We can then directly compare the Green-Kubo results obtained from an atomic configuration stabilized at $10 \mathrm{~K}$, with the results of our calculation from the same atomic configuration, but taken at high temperature for having the same classical phonon population. This comparison is reported in Fig. 7 , and shows a very good agreement for amorphous and nanocomposites with a volume fraction of $30 \%$. We can clearly see the effect of the elastic contrast on the calculated thermal conductivity, however it is smaller than the error bars of the Green-Kubo calculations, explaining thus the apparent insensitivity of these latter.

To get more insight on the effect of the elastic contrast on the two different thermal transport regimes, we report in Fig. 9 the two contributions as a function of temperature. The high temperature saturation of the propagative contribution is clearly due to the absence of contributing modes above Ioffe-Regel: the saturation temperature corresponds to $T_{I R} \approx \hbar \omega_{I R} / k_{B}$ and is clearly reduced in nanocomposites with respect to the amorphous. It is evident that nanostructuration causes a reduction of the propagative part, stronger in presence of a harder inclusion. As for the diffusive part, this is enhanced by the presence of the nanoinclusions, 


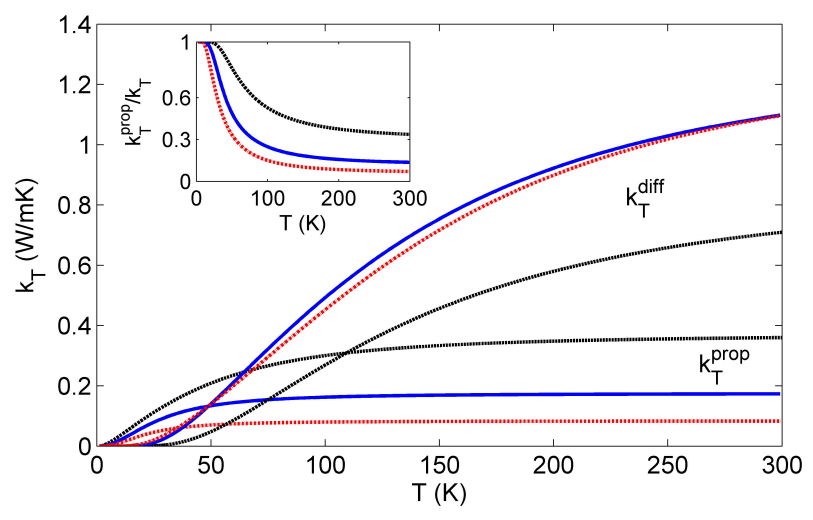

Fig. 9 Propagative and Diffusive contributions to the thermal conductivity calculated using Eqs. 10 and 11 as a function of temperature for the amorphous system (black dashed line), and two composite systems with $\chi=1$ (blue solid line) and $\chi=4.6$ (red dot-dashed line) at a volume fraction $\phi=30 \%$. Inset: Relative weight of the propagative contribution, same colors and line styles as the main figure.

with almost no effect of the elastic contrast. Such enhancement is due to a major interface scattering and the increased number of diffusive modes, as indicated by the drastic reduction of the Ioffe-Regel crossover. At the end, due to the reduction of the propagative phase space, if at low temperature propagons dominate heat transport, at room temperature they only account for about $10 \%$ of it in the harder nanocomposite, against $30 \%$ for the amorphous sample (see inset of the figure).

\section{Conclusions}

In this work we have reported a microscopic investigation of phonon dynamics in amorphous/nanocrystalline composites, with the aim of evidencing and understanding the role of elastic contrast between matrix and nanoinclusions. As cited above, the elastic contrast is expected to play a major role in determining thermal transport properties in such nanocomposites. Still, contradictory experimental results have been reported.

Here we have shown that the presence of a crystalline nanoinclusion modifies phonon dynamics even in absence of an elastic contrast. Still, the effect is much stronger when the nanoinclusion is harder than the matrix. The presence of a disorder/order interface, coupled with the elastic heterogeneity, is at the origin of a modification in acoustic wave-packets, which loose their propagative character, leading to a significant anticipation of the Ioffe-Regel crossover between a propagative and a diffusive regime. Such anticipation translates in several modifications of the microscopic dynamics, from the breaking down of the individual phonon in an envelope of modes, to the rising up of a low energy peak in the reduced phonon density of states, at energies below the Boson Peak. The inspection of the time evolution of longitudinal and transverse wave-packets generated in the sample clearly confirms such anticipation and the arising of a strongly inhomogeneous energy propagation in presence of elastic contrast, with the pinning of vibrational energy within the harder inclusions. An anticipated Ioffe-Regel crossover means that the transition from a ballistic to a diffusive thermal transport takes place at lower frequencies, reducing the phase space for propagons to about half the one in the fully amorphous system for the high contrast nanocomposite. Despite such a strong effect on acoustic modes, the thermal conductivity, as calculated with the Green-Kubo formula, is almost unaffected. This is essentially due to the major diffusive nature of thermal transport already in the amorphous matrix when all phonons are thermally populated. The dramatic reduction of the propagative contribution affects thus only partially the total thermal conductivity, being balanced by the increased diffusive contribution in the nanocomposites, which, moreover, is quite insensitive to the elastic contrast.

Interestingly, the interface scattering dominates thermal conductivity only for the atomic configuration equilibrated at low temperature, where it counterbalances the larger crystalline contribution. At room temperature however, this is not true anymore: the higher conductivity in the crystalline nanoinclusions takes it over the interface scattering, whose strength decreases indeed with temperature, assuring a gradual increase of the thermal conductivity with crystalline volume fraction, resulting in a behavior in agreement with effective medium predictions.

Our findings, obtained on a model system, allow to clarify the panoply of experimental and simulation results on thermal conductivity in systems with and without elastic contrast. We find indeed that this latter is a key ingredient, dramatically perturbing phonon dynamics, and reducing the propagative contribution to thermal transport. Such reduction is due to the strong phonon scattering introduced with the nanostructuration, which leads to two consequences: the reduction of the phonon mean free path, and the anticipation of the Ioffe-Regel crossover, reducing thus the phase space of propagative phonons and then the number of modes available for a propagative thermal transport. However, the effect of such modifications of the microscopic dynamics on the macroscopic thermal conductivity is not straightforward. The result will depend on the relative weight of propagative and diffusive contribution to thermal transport in the matrix material. If propagons are the main heat carriers, a strong reduction of the thermal conductivity can be expected. If diffusive transport dominates already in the matrix, then no reduction is expected, and the thermal conductivity could even increase, as the diffusive contribution increases. Depending on this crucial point - which will depend on the material and on temperature - the sensitivity of thermal conductivity to elastic contrast will strongly change as well. Our findings clearly indicate that an amorphous matrix where propagons carry most of the heat at room temperature would be better suited for enhancing the interface scattering and elastic contrast effect and ultimately engineering nanocomposites with even lower thermal conductivity than the matrix. Our study illustrates as well the relevance of coupling a microscopic dynamics investigation to thermal conductivity simulations to get a real insight onto the mechanisms at play and the key parameters for thermal transport in heterogeneous systems. 


\section{Conflicts of interest}

There are no conflicts to declare.

\section{Acknowledgement}

The authors are grateful to Région Rhône-Alpes who has funded this work through the ARC Energie PhD funding program. V. M. G. thanks the Lyon IDEX Scientific Breakthrough program for funding of the project IPPON. The work of Y.M.B. was supported by the Russian Federation President Grant no. MK-3052.2019.2 and the 2018 Metchnikov fellowship of the French Embassy in Russia.

\section{Notes and references}

1 D. o. E. Lawrence Livermore National Laboratory, Data based on DOE/EIA MER (2017), 2017, https://flowcharts. llnl.gov/commodities/energy.

2 Z. Viskadourakis, G. Perrakis, E. Symeou, J. Giapintzakis and G. Kenanakis, Applied Physics A: Materials Science \& Processing, 2019, 125, 159.

3 O. Bourgeois, D. Tainoff, A. Tavakoli, Y. Liu, C. Blanc, M. Boukhari, A. Barski and E. Hadji, C. R. Physique, 2016, 17, 1154 .

4 Y. Nakamura, M. Isogawa, T.Ueda, S. Yamasaka, H. Matsui, J. Kikkawa, S.Ikeuchi, T.Oyake, T. Hori, J. Shiomi and A. Sakai, Nano Energy, 2015, 12, 845.

5 M. Verdier, K. Termentzidis and D. Lacroix, Journal of Applied Physics, 2016, 119, 175104.

6 S. Volz, J. Shiomi, M. Nomura and K. Miyazaki, Journal of Thermal Science and Technology, 2016, 11, year.

7 M. Jeng, R. Yang, D. Song and G. Chen, Journal of Heat Transfer, 2008, 130, 042410-1.

8 F. B. Juangsa, Y. Muroya, M. Ryu, J. Morikawa and T. Nozaki, J. Phys. D : Appl. Phys., 2016, 49, 365303.

9 F. B. Juangsa, Y. Muroya, M. Ryu, J. Morikawa and T. Nozaki, Appl. Phys. Lett., 2017, 110, 253105.

10 A. Minnich and G. Chen, Appl. Phys. Lett., 2007, 91, 073105.

11 J. Ordonez-Miranda, R. Yang and J. J. Alvarado-Gil, Appl. Phys. Lett., 2011, 98, 233111.

12 L. Yang and A. Minnich, Scientific Reports, 2017, 7, year.

13 T. Damart, V. M. Giordano and A. Tanguy, Physical Review B, 2015, 92, 094201.

14 A. Tlili, S. Pailhès, R. Debord, B. Ruta, S. Gravier, J.-J. Blandin, N. Blanchard, S. Gomès, A. Assy, A. Tanguy and V. M. Giordano, Acta Materialia, 2017, 136, 425.

15 K. Termentzidis, V. M. Giordano, M. Katsikini, E. Paloura, G. Pernot, D. Lacroix, T. Karakostas and J. Kioseoglou, Nanoscale, 2018, 10, 21732-21741.

16 Y. Beltukov, C. Fusco, D. Parshin and A. Tanguy, Phys. Rev. E, 2016, 93, 023006.

17 A. Tanguy, J. Wittmer, F. Léonforte and J.-L. Barrat, Physical Review B, 2002, 66, 174205.

18 P. B. Allen, J. L. Feldman, J. Fabian and F. Wooten, Philosoph- ical Magazine B, 1999, 79, 1715.

19 P. B. Allen and J. L. Feldman, Physical Review B, 1993, 48, 12581.

20 J. Ludman, S. Taraskin and S. Elliott, Physical Review B, 2003, 67, 132203.

21 J. Tersoff, Phys Rev B, 1988, 37, 6991.

22 C. Fusco, T. Albaret and A. Tanguy, Phys. Rev. E, 2010, 82, 066116.

23 F. Stillinger and T. Weber, Phys Rev B, 1985, 31, 5262.

24 L. Pizzagalli, J. Godet, J. Guénolé, S. Brochard, E. Holmstrom, K. Nordlund and T. Albaret, Journal of Physics: Condensed Matter, 2013, 25, 055801.

25 A. Tlili, MSc thesis, Ecole Doctorale Matŕiaux - Université Lyon 1, 2017.

26 A. Weisse, G. Wellein, A. Alvermann and H. Fehske, Rev. Mod. Phys., 2006, 78, 275.

27 Y. M. Beltukov, D. A. Parshin, V. M. Giordano and A. Tanguy, Phys. Rev. E, 2018, 98, 023005.

28 J. Che, T. Cagin, W. Deng and W. Goddard, J. Chem. Phys., 2000, 113, 6888.

29 P. Schelling, S. Phillpot and P. Keblinski, Phys. Rev. B, 2002, 65, 144306.

30 K. Termentzidis, S. Merabia, P. Chantrenne and P. Keblinski, International Journal of Heat and Mass Transfer, 2011, 54, 2014.

31 A. France-Lanord, S. Merabia, T. Albaret, D. Lacroix and K. Termentzidis, J. Phys.: Condens. Matter, 2014, 26, 355801.

32 J. Barrat and F. Chiaruttini, Mol. Phys., 2003, 101, 16051610.

33 K. Esfarjani, G. Chen and H. Stokes, Phys. Rev. B, 2012, 84, 085204.

34 T. Damart, A. Tanguy and D. Rodney, Phys Rev B, 2017, 95, 054203.

35 B. Qi, J. Absi and N.Tessier-Doyen, Computational Materials Science, 2009, 46, 996.

36 S. Torquato, Random heterogeneous materials: microstructure and macroscopic properties., Springer Science \& Business Media, 2013.

37 D. F. Swinehart, Journal of Chemical Education, 1962, 39, 333.

38 J. M. Larkin and A. J. H. McGaughey, Phys. Rev. B, 2014, 89, 144303.

39 W. Lv and A. Henry, New J. Phys, 2016, 18, 013028.

40 Y. He, D. Donadio and G. Galli, Applied Physics Letters, 2011, 98, 144101.

41 S.Ju and X. Liang, J. Appl. Phys, 2012, 112, 064305.

42 D. Checke and H. Ettinger, Journal of Low Temperatures Physics, 1979, 36, 121.

43 W. Eisenmenger, in Phonon Scattering in Condensed Matter V, ed. A. C. Anderson and J. P. Wolfe, Springer, 1986, ch. Phonon Scattering at Surfaces and Interfaces., p. 204. 